CRYSTALLOGRAPHIC COMMUNICATIONS

ISSN 2056-9890

Received 5 December 2016

Accepted 2 February 2017

Edited by M. Weil, Vienna University of Technology, Austria

Keywords: crystal structure; borate; spontaneous nucleation; solid solution.

CCDC reference: 1530747

Supporting information: this article has supporting information at journals.iucr.org/e

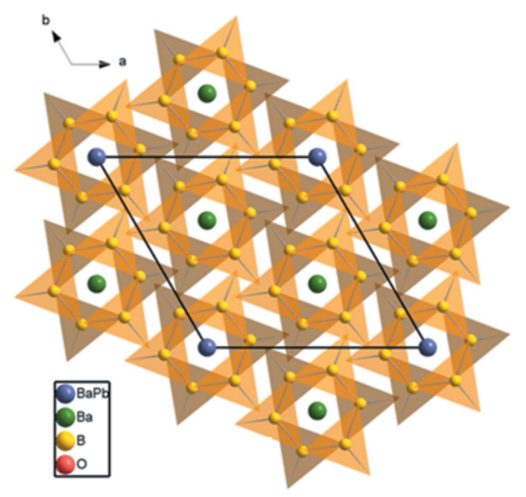

OPEN $\odot$ ACCESS

\section{Crystal structure of the solid solution $\mathrm{Ba}_{8.35} \mathrm{~Pb}_{0.65}\left(\mathrm{~B}_{3} \mathrm{O}_{6}\right)_{6}$}

\author{
Wenwu Zhao*
}

Department of Environmental and Chemical Engineering, Tangshan College, 38 North HuaYan Road, Tangshan 063000, Hebei, People's Republic of China. *Correspondence e-mail: zww995@163.com

Single crystals of lead barium borate, $\mathrm{Ba}_{8.35} \mathrm{~Pb}_{0.65}\left(\mathrm{~B}_{3} \mathrm{O}_{6}\right)_{6}$, octabarium lead(II) hexakis(triborate), have been obtained by spontaneous nucleation from a hightemperature melt. Its three-dimensional structure is constructed on the basis of $\mathrm{a}^{\mathrm{BaO}} 9$ polyhedron, a $(\mathrm{Pb} / \mathrm{Ba}) \mathrm{O}_{6}$ octahedron (occupancy ratio $\mathrm{Pb}: \mathrm{Ba}=$ 0.216:0.784) and a condensed $\mathrm{B}_{3} \mathrm{O}_{6}$ ring anion. In the crystal, the planar $\mathrm{B}_{3} \mathrm{O}_{6}$ anions are stacked in an alternating fashion with $\mathrm{Ba}$ and $(\mathrm{Pb} / \mathrm{Ba})$ atoms along [001]. A comparison is made with the structures of related solid solutions in the system $\mathrm{Ba} / \mathrm{Pb} / \mathrm{B} / \mathrm{O}$.

\section{Chemical context}

The study of inorganic borates is motivated by their possible non-linear optical properties, transparency in a wide range of wavelengths, high laser-damage tolerance, piezoelectricity and luminescent and other useful properties for technical applications of the respective compounds. For example, $\beta-\mathrm{BaB}_{2} \mathrm{O}_{4}$ (Chen et al., 1985), $\mathrm{LiB}_{3} \mathrm{O}_{5}$ (Chen et al., 1989), $\mathrm{CsB}_{3} \mathrm{O}_{5}$ (Sasaki et al., 2000), $\mathrm{Sr}_{2} \mathrm{Be}_{2} \mathrm{~B}_{2} \mathrm{O}_{7}$ (Chen et al., 1995), $\mathrm{K}_{5} \mathrm{Ba}_{10}\left(\mathrm{BO}_{3}\right)_{8} \mathrm{~F}$ (Liu et al., 2016), $\mathrm{PbB}_{4} \mathrm{O}_{7}$ (Bartwal et al., 2001), $\mathrm{Pb}_{2} \mathrm{~B}_{5} \mathrm{O}_{9} X$ ( $X=\mathrm{Cl}, \mathrm{Br}, \mathrm{I}$ ) (Huang et al., 2010) or $\mathrm{Ba}_{3} \mathrm{Sr}_{4}\left(\mathrm{BO}_{3}\right)_{3} \mathrm{~F}_{5}$ (Zhang et al., 2009) have been studied because of their second-order

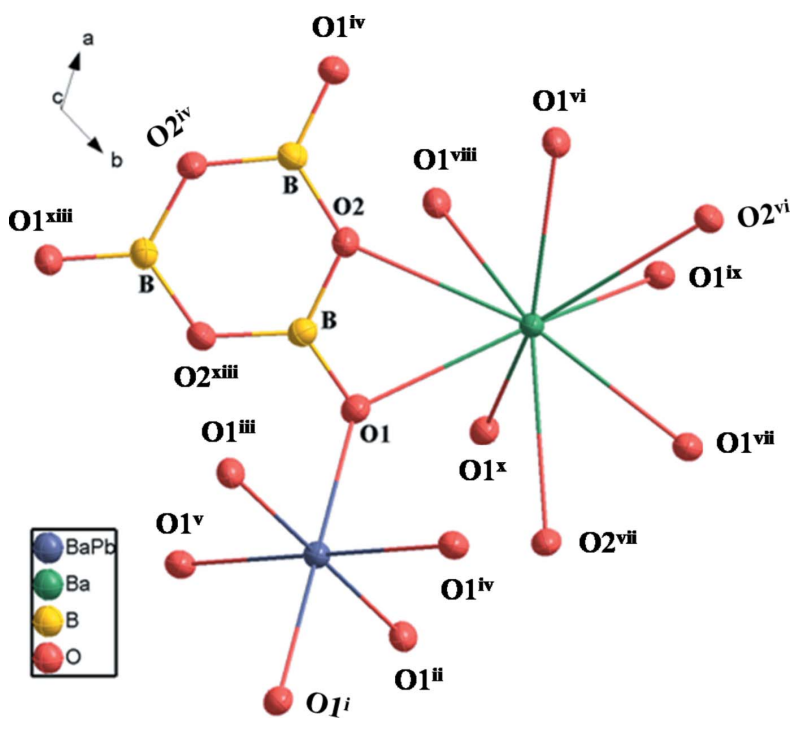

Figure 1

The principal building units in the crystal structure of the title compound. Displacement ellipsoids are drawn at the $50 \%$ probability level. [Symmetry codes: (i) $-x,-y,-z$; (ii) $-y, x-y, z$; (iii) $y,-x+y,-z$; (iv) $x-y, x,-z$; (v) $-x+y,-x$, $z$; (vi) $-y+1, x-y$, $z$; (vii) $-x+y+1$, $-x+1, z$; (viii) $y+\frac{2}{3},-x+y+\frac{1}{3},-z+\frac{1}{3}$; (ix) $x-y+\frac{2}{3}, x+\frac{1}{3},-z+\frac{1}{3}$; (x) $-x+\frac{2}{3},-y+\frac{1}{3},-z+\frac{1}{3}$; (xiii) $-y, x-y-1, z ;$ (xiv) $-x+y+1,-x, z$.] 
non-linear optical behavior. Among inorganic borates synthesized and characterized over the past decades, some lead(II) borates show comprehensive applications. These features are associated with the highly asymmetric stereochemistry typical for a lead(II) atom due to the stereoactivity of the $6 s^{2}$ lone pair (Zhang et al., 2016; Mutailipu et al., 2016). Accordingly, numerous studies have been devoted to this family of compounds. Some lead borates are particularly attractive because of their high second-harmonic generation (SHG) response (Wu et al., 2012; Dong et al., 2015; Jing et al., 2015) or large birefringence (Liu et al., 2015).

In this communication, we report on the synthesis and crystal structure of the solid solution $\mathrm{Ba}_{8.35} \mathrm{~Pb}_{0.65}\left(\mathrm{~B}_{3} \mathrm{O}_{6}\right)_{6}$.

\section{Structural commentary}

The crystal structure of $\mathrm{Ba}_{8.35} \mathrm{~Pb}_{0.65}\left(\mathrm{~B}_{3} \mathrm{O}_{6}\right)_{6}$ is based on a $\mathrm{Ba}_{2} \mathrm{O}_{9}$ polyhedron, a $(\mathrm{Pb} / \mathrm{Ba} 1) \mathrm{O}_{6}$ polyhedron and $\mathrm{a}$ condensed $\mathrm{B}_{3} \mathrm{O}_{6}$ anion, as shown in Fig. 1. The planar $\mathrm{B}_{3} \mathrm{O}_{6}$ anions (point group symmetry 3.) are isolated from each other and distributed layer upon layer perpendicular to [001]. The occupationally disordered $(\mathrm{Pb} / \mathrm{Ba}) 1$ site (occupancy ratio $\mathrm{Pb}: \mathrm{Ba}=0.216: 0.784)$ and the $\mathrm{Ba} 2$ site are located alternately between the $\mathrm{B}_{3} \mathrm{O}_{6}$ sheets in $(\mathrm{Pb} / \mathrm{Ba}) 1$ and $\mathrm{Ba} 2$ layers, as shown in Fig. $2 a$. The $\mathrm{B}$ atom is bound to one $\mathrm{O} 1$ atom and two $\mathrm{O} 2$ atoms to from a $\mathrm{BO}_{3}$ triangle. Three $\mathrm{BO}_{3}$ triangles are condensed through vertex-sharing to build a planar and cyclic $\mathrm{B}_{3} \mathrm{O}_{6}$ unit. The $\mathrm{B}-\mathrm{O}$ bond lengths vary from 1.318 (5) to

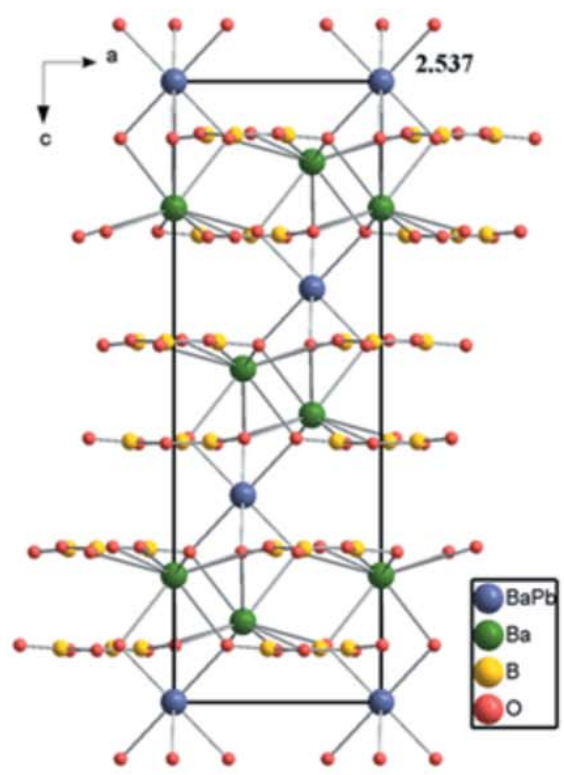

(a)

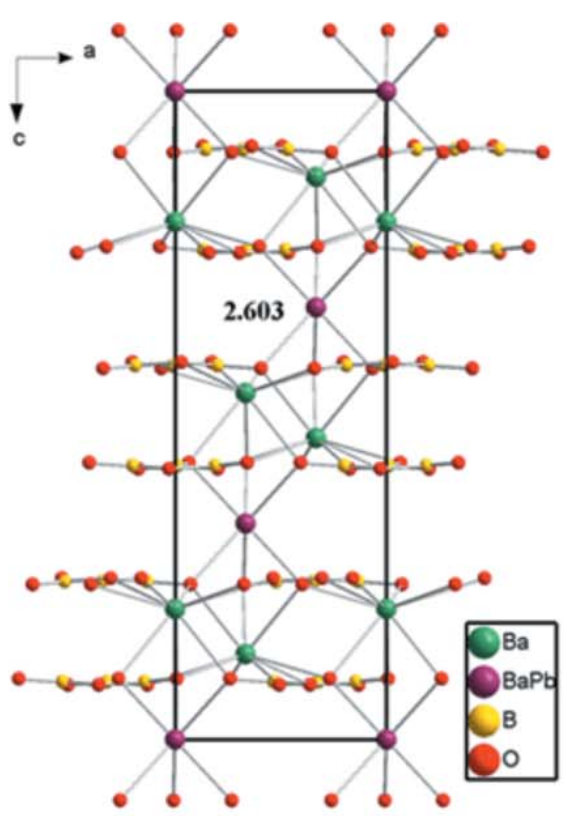

(b)

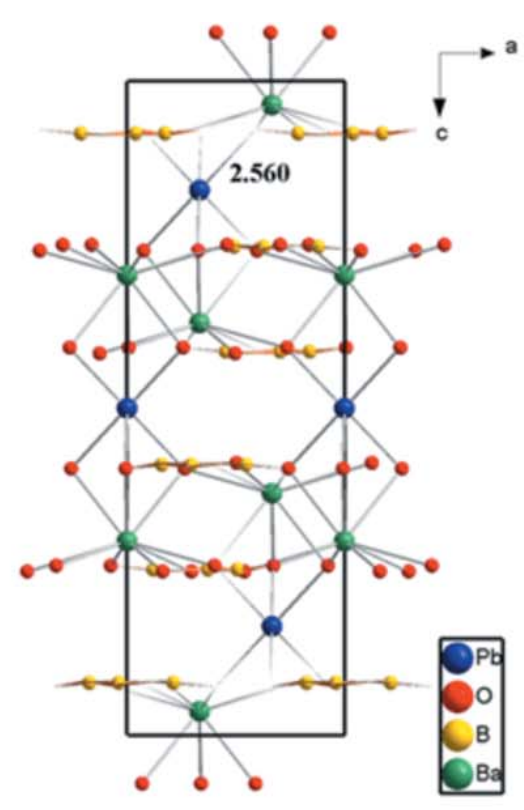

(c)

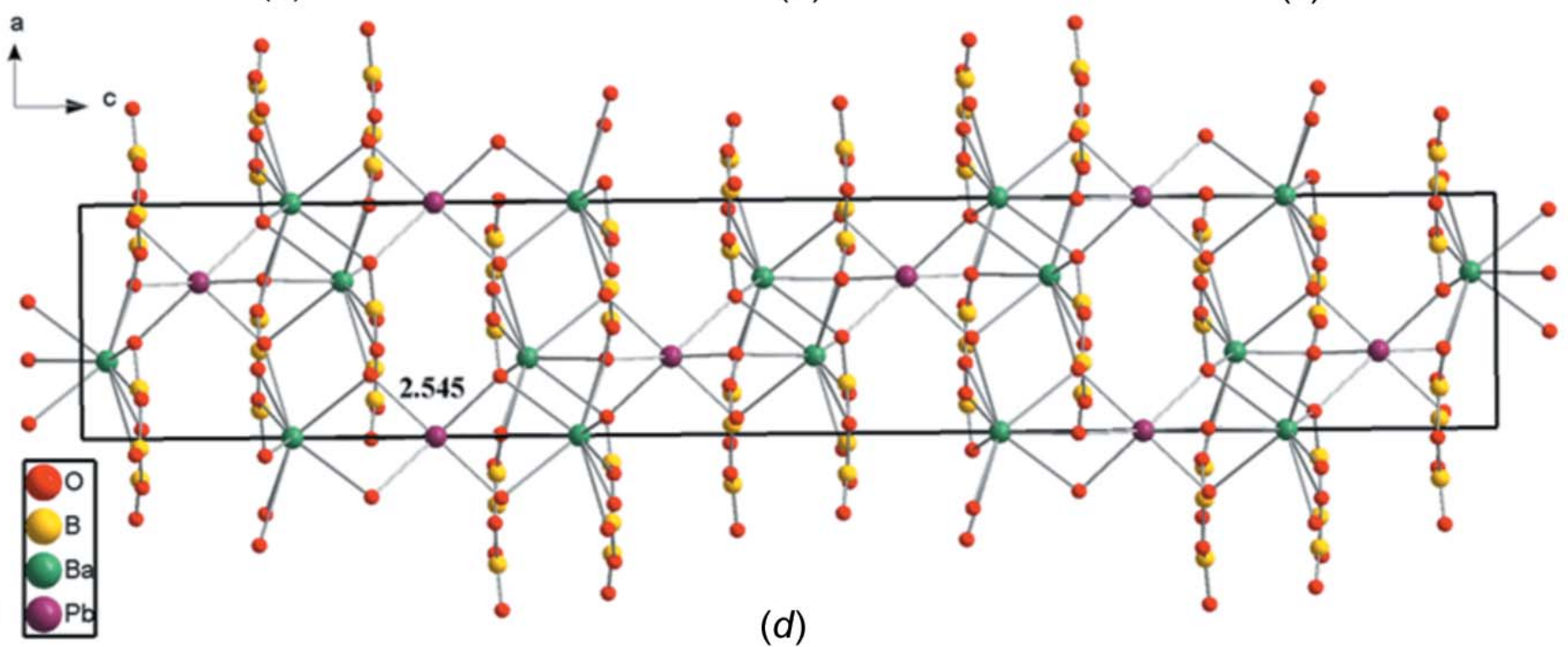

Figure 2

The crystal structures of related solid solutions in the system $\mathrm{Ba} / \mathrm{Pb} / \mathrm{B} / \mathrm{O}$ viewed down [010]: $(a) \mathrm{Ba}_{8.35} \mathrm{~Pb}_{0.65}\left(\mathrm{~B}_{3} \mathrm{O}_{6}\right)_{6} ;\left(\right.$ b) $\mathrm{Ba}_{7.87} \mathrm{~Pb}_{1.13}\left(\mathrm{~B}_{3} \mathrm{O}_{6}\right)_{6}\left(\mathrm{Wu}_{\text {e }}\right.$ et al. , 2012); (c) $\mathrm{Ba}_{2} \mathrm{~Pb}\left(\mathrm{~B}_{3} \mathrm{O}_{6}\right)_{2}$ (Li et al., 2014); (d) $\mathrm{Ba}_{2} \mathrm{~Pb}\left(\mathrm{~B}_{3} \mathrm{O}_{6}\right)_{2}$ (Tang et al., 2015). The numbers indicate the bond lengths $(\AA)$ of the $\mathrm{PbO}_{6}$ or $(\mathrm{Ba} / \mathrm{Pb}) \mathrm{O}_{6}$ octahedra. 


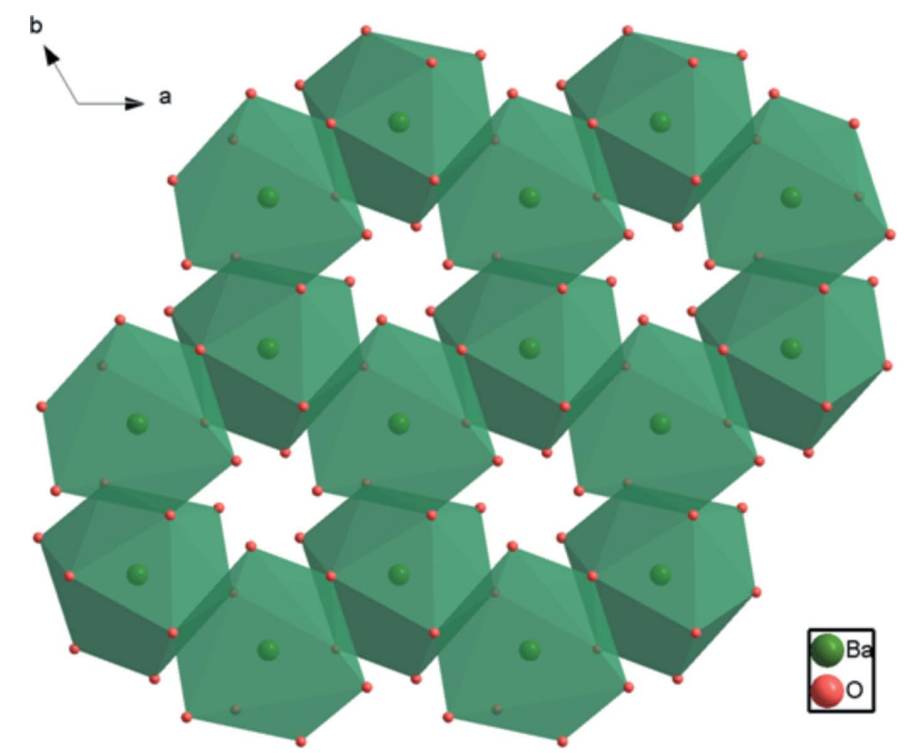

Figure 3

The formation of a corrugated layer of $\mathrm{Ba}_{2} \mathrm{O}_{9}$ polyhedra in the crystal structure of $\mathrm{Ba}_{8.35} \mathrm{~Pb}_{0.65}\left(\mathrm{~B}_{3} \mathrm{O}_{6}\right)_{6}$ viewed down [001].

1.406 (5) $\AA$ (Table 1), and the $\mathrm{O}-\mathrm{B}-\mathrm{O}$ angles are between $116.8(4)$ and $122.6(4)^{\circ}$.
Table 1

Selected geometric parameters $\left(\AA{ }^{\circ}{ }^{\circ}\right)$.

\begin{tabular}{lrll}
\hline$(\mathrm{Pb} / \mathrm{Ba}) 1-\mathrm{O} 1$ & $2.537(3)$ & $\mathrm{B}-\mathrm{O} 1$ & $1.318(5)$ \\
$\mathrm{Ba} 2-\mathrm{O} 1$ & $2.766(3)$ & $\mathrm{B}-\mathrm{O} 2$ & $1.397(5)$ \\
$\mathrm{Ba} 2-\mathrm{O} 1^{\mathrm{i}}$ & $2.810(3)$ & $\mathrm{B}-\mathrm{O} 2^{\mathrm{ii}}$ & $1.406(5)$ \\
$\mathrm{Ba} 2-\mathrm{O} 2$ & $3.030(3)$ & & \\
& & & \\
$\mathrm{O} 1-\mathrm{B}-\mathrm{O} 2$ & $120.6(4)$ & $\mathrm{O} 2-\mathrm{B}-\mathrm{O}^{\mathrm{ii}}$ & $116.8(4)$ \\
$\mathrm{O} 1-\mathrm{B}-\mathrm{O} 2^{\mathrm{ii}}$ & $122.6(4)$ & & \\
\hline
\end{tabular}

Symmetry codes: (i) $-x+\frac{2}{3},-y+\frac{1}{3},-z+\frac{1}{3}$; (ii) $-y, x-y-1, z$.

The $\mathrm{Ba} 2$ atom (site symmetry 3.) is coordinated by nine $\mathrm{O}$ atoms. The $\mathrm{Ba}-\mathrm{O}$ bond lengths of the $\mathrm{Ba}_{2} \mathrm{O}_{9}$ polyhedron range from 2.766 (3) to 3.030 (3) $\AA$, with a mean distance of $2.869 \AA$ (Table 1). A similar environment for $\mathrm{Ba}$ is observed in the crystal structures of $\mathrm{Na}_{3} \mathrm{Ba}_{2}\left(\mathrm{~B}_{3} \mathrm{O}_{6}\right)_{2} \mathrm{~F}$ (Zhang et al., 2015), $\mathrm{PbBa}_{2}\left(\mathrm{~B}_{3} \mathrm{O}_{6}\right)_{2}$ (Li et al., 2014) and $\alpha$-BBO (Wu et al., 2002). Each of the $\mathrm{Ba}_{2} \mathrm{O}_{9}$ polyhedra shares edges with adjacent $\mathrm{Ba}_{2} \mathrm{O}_{9}$ polyhedra to form six-membered rings that are arranged in corrugated layers extending parallel to (001) (Fig. 3). The $(\mathrm{Pb} / \mathrm{Ba}) 1$ site ( site symmetry $\overline{3}$.) is surrounded by six $\mathrm{O}$ atoms; the corresponding $(\mathrm{Pb} / \mathrm{Ba}) 1 \mathrm{O}_{6}$ octahedra are isolated from each other. The six $(\mathrm{Pb} / \mathrm{Ba} 1)-\mathrm{O}$ bonds have an identical length of 2.537 (3) ^ (Table 1, Fig. 2a). In comparison with the $M 2$ site, the $M 1$ site has a more narrow

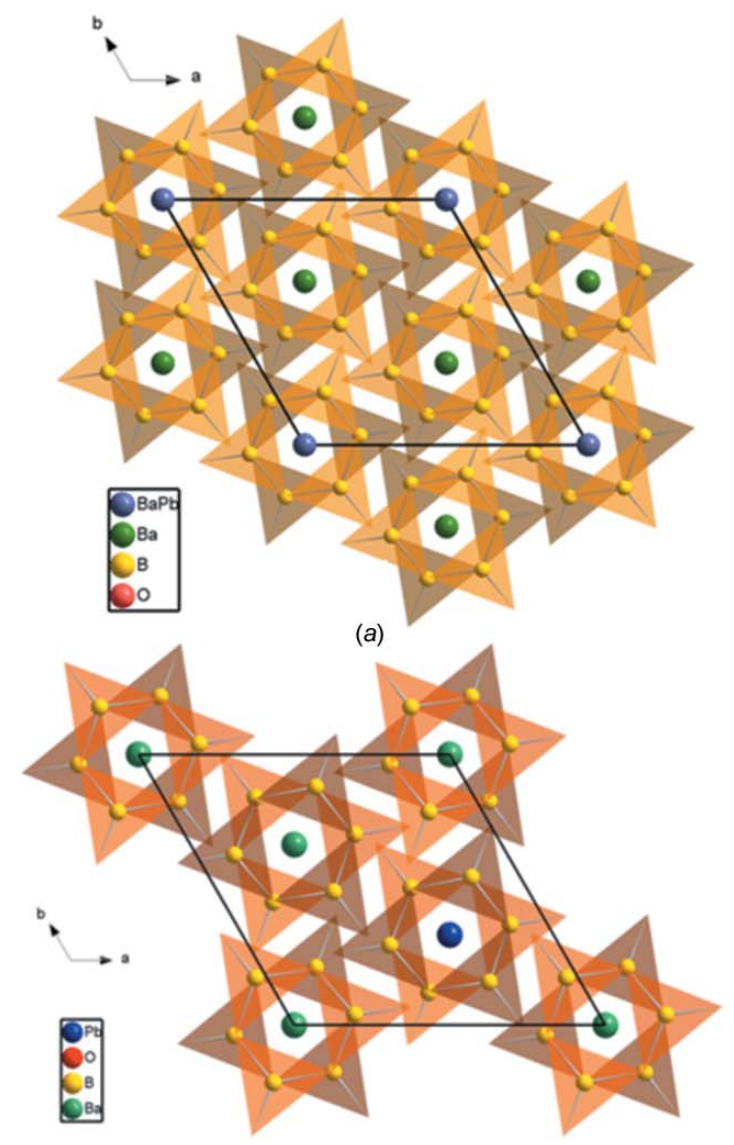

(c)

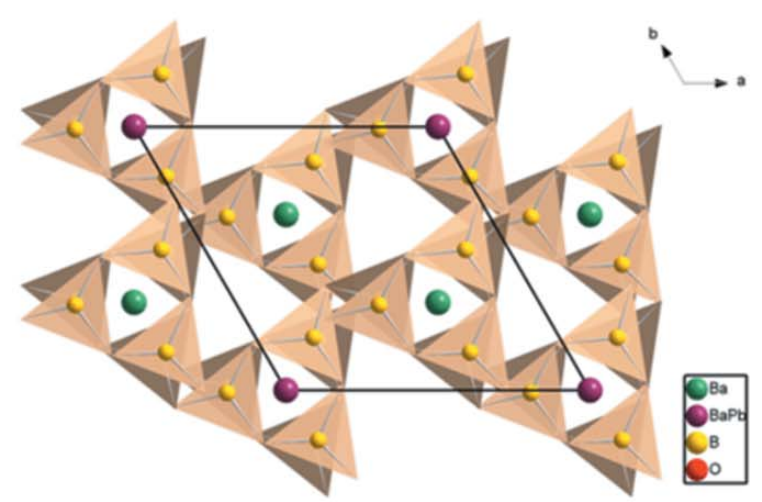

(b)

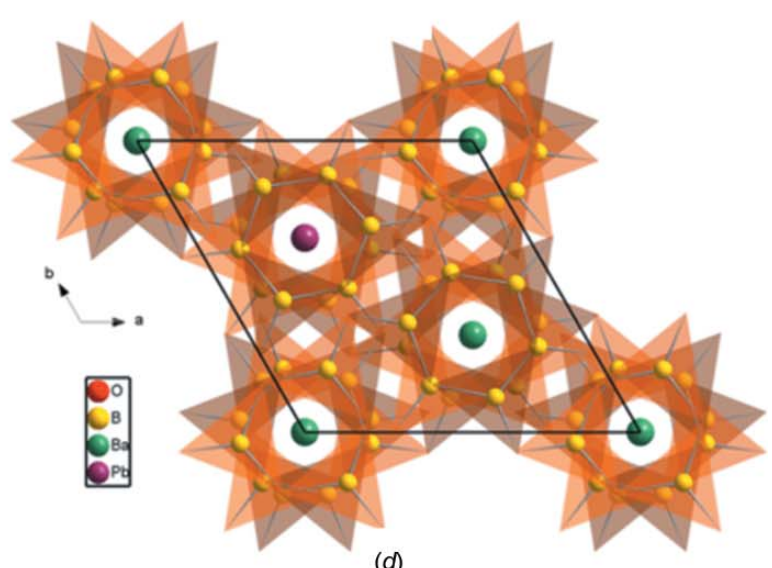

Figure 4

The arrangement of $\mathrm{B}_{3} \mathrm{O}_{6}$ groups along the [001] direction in the different solid solutions: $(a) \mathrm{Ba}_{8.35} \mathrm{~Pb}_{0.65}\left(\mathrm{~B}_{3} \mathrm{O}_{6}\right)_{6} ;(b) \mathrm{Ba}_{7.87} \mathrm{~Pb}_{1.13}\left(\mathrm{~B}_{3} \mathrm{O}_{6}\right)_{6}\left(\mathrm{Wu}_{\text {et }}\right.$ al. , 2012); (c) $\mathrm{Ba}_{2} \mathrm{~Pb}\left(\mathrm{~B}_{3} \mathrm{O}_{6}\right)_{2}$ (Li et al., 2014); (d) $\mathrm{Ba}_{2} \mathrm{~Pb}\left(\mathrm{~B}_{3} \mathrm{O}_{6}\right)_{2}$ (Tang et al., 2015). 
coordination environment which seems to be the reason why $\mathrm{Pb}$ atoms exclusively substitute $\mathrm{Ba}$ atoms at the latter position due to their smaller ionic radius.

\section{Comparison with the structures of related solid solutions}

It is interesting to compare the structure of $\mathrm{Ba}_{8.35} \mathrm{~Pb}_{0.65}\left(\mathrm{~B}_{3} \mathrm{O}_{6}\right)_{6}$ with those of the related solid solutions $\mathrm{Ba}_{7.87} \mathrm{~Pb}_{1.13}\left(\mathrm{~B}_{3} \mathrm{O}_{6}\right)_{6}$ (Wu et al., 2012) and $\mathrm{Ba}_{2} \mathrm{~Pb}\left(\mathrm{~B}_{3} \mathrm{O}_{6}\right)_{2}$ (Li et al., 2014; Tang et al., $2015)$. Whereas the title compound $\mathrm{Ba}_{8.35} \mathrm{~Pb}_{0.65}\left(\mathrm{~B}_{3} \mathrm{O}_{6}\right)_{6}$ crystallizes in space group $R \overline{3}, \mathrm{Ba}_{7.87} \mathrm{~Pb}_{1.13}\left(\mathrm{~B}_{3} \mathrm{O}_{6}\right)_{6}$ was solved and refined in space group $R 32$ on the basis of single crystal X-ray diffraction data (Wu et al., 2012); the lattice parameters of both compounds are very similar. $\mathrm{Ba}_{2} \mathrm{~Pb}\left(\mathrm{~B}_{3} \mathrm{O}_{6}\right)_{2}$ on the other hand was reported to crystallize either in space group $R \overline{3}$ with lattice parameters in the same range as the previous two structures (single crystal X-ray diffraction data; Li et al., 2014) or in space group $R \overline{3} c$ with a doubled $c$ axis in comparison with the other structures (powder X-ray diffraction data using the Rietveld method; Tang et al., 2015). All four crystal structures are characterized by an alternating stacking of cationic and anionic (001) layers along [001], as shown in Fig. 2. In each case, the $\mathrm{Ba}$ site is coordinated by nine $\mathrm{O}$ atoms to form $\mathrm{BaO}_{9}$ polyhedra, and the $\mathrm{Pb}$ or the $(\mathrm{Pb} / \mathrm{Ba})$ site is coordinated by six $\mathrm{O}$ atoms to form distorted $\mathrm{PbO}_{6}$ octahedra [in $\mathrm{Ba}_{2} \mathrm{~Pb}\left(\mathrm{~B}_{3} \mathrm{O}_{6}\right)_{2}$ ] or $(\mathrm{Pb} / \mathrm{Ba}) \mathrm{O}_{6}$ octahedra $\left[\right.$ in $\mathrm{Ba}_{8.35} \mathrm{~Pb}_{0.65}\left(\mathrm{~B}_{3} \mathrm{O}_{6}\right)_{6}$ and $\left.\mathrm{Ba}_{7.87} \mathrm{~Pb}_{1.13}\left(\mathrm{~B}_{3} \mathrm{O}_{6}\right)_{6}\right]$.

The arrangement of the planar $\mathrm{B}_{3} \mathrm{O}_{6}$ rings in the crystal structures is a determining factor in whether a non-centrosymmetric or a centrosymmetric structure is obtained. In $\mathrm{Ba}_{7.87} \mathrm{~Pb}_{1.13}\left(\mathrm{~B}_{3} \mathrm{O}_{6}\right)_{6}(\mathrm{Wu}$ et al., 2012), the rings are aligned in a chiral arrangement (Fig. $4 b$ ), responsible for the SHG effect. In $\mathrm{Ba}_{2} \mathrm{~Pb}\left(\mathrm{~B}_{3} \mathrm{O}_{6}\right)_{2}$ ( $\mathrm{Li}$ et al., 2014), the $\mathrm{B}_{3} \mathrm{O}_{6}$ rings are parallel to each other, distributed layer upon layer along [001], and the $\mathrm{B}_{3} \mathrm{O}_{6}$ rings in neighbouring layers point in exactly opposite directions (Fig. 4c), just like in the title compound (Fig. 4a). In the $\mathrm{Ba}_{2} \mathrm{~Pb}\left(\mathrm{~B}_{3} \mathrm{O}_{6}\right)_{2}$ structure with doubled volume (Tang et al., 2015), all of the $\mathrm{B}_{3} \mathrm{O}_{6}$ rings are parallel to (001), and the $\mathrm{B}_{3} \mathrm{O}_{6}$ rings in two neighbouring layers are rotated slightly relative to each other (Fig. $4 d$ ).

\section{Synthesis and crystallization}

Suitable crystals of the solid solution $\mathrm{Ba}_{8.35} \mathrm{~Pb}_{0.65}\left(\mathrm{~B}_{3} \mathrm{O}_{6}\right)_{6}$ were obtained by spontaneous nucleation from a high-temperature melt mixture originating from $\mathrm{PbO}, \mathrm{H}_{3} \mathrm{BO}_{3}$ and $\mathrm{BaF}_{2}$ in molar ratios of 4:5:1. The starting materials were weighed and melted in a platinum crucible in several batches. The crucible position was fixed at the centre of a resistance-heated furnace. The temperature of the furnace was controlled within $0.1-1 \mathrm{~K}$ by an Al-708P controller and a $\mathrm{Pt} / \mathrm{Pt}-\mathrm{Rh}$ thermocouple. The temperature was raised by about $50 \mathrm{~K} \mathrm{~h}^{-1}$ to $50 \mathrm{~K}$ above the melting point and held for $15 \mathrm{~h}$ to ensure a homogenous mixture of the solution. After cooling down the furnace to $1073 \mathrm{~K}$, a slow cooling rate of $5 \mathrm{~K} \mathrm{~d}^{-1}$, was applied, followed
Table 2

Experimental details.

\begin{tabular}{|c|c|}
\hline \multicolumn{2}{|l|}{ Crystal data } \\
\hline Chemical formula & $\mathrm{Ba}_{8.35} \mathrm{~Pb}_{0.65}\left(\mathrm{~B}_{3} \mathrm{O}_{6}\right)_{6}$ \\
\hline$M_{\mathrm{r}}$ & 2051.83 \\
\hline Crystal system, space group & Trigonal, $R \overline{3}$ \\
\hline Temperature $(\mathrm{K})$ & $296^{\circ}$ \\
\hline$a, c(\AA)$ & $7.206(2), 18.653$ (11) \\
\hline$V\left(\AA^{3}\right)$ & $838.7(6)$ \\
\hline$Z$ & 1 \\
\hline Radiation type & Мо $K \alpha$ \\
\hline$\mu\left(\mathrm{mm}^{-1}\right)$ & 13.00 \\
\hline Crystal size $(\mathrm{mm})$ & $0.16 \times 0.08 \times 0.02$ \\
\hline \multicolumn{2}{|l|}{ Data collection } \\
\hline Diffractometer & Bruker APEXII CCD \\
\hline Absorption correction & $\begin{array}{l}\text { Numerical (face-indexed using } \\
\quad S A D A B S ; \text { Bruker, 2000) }\end{array}$ \\
\hline$T_{\min }, T_{\max }$ & $0.141,0.547$ \\
\hline $\begin{array}{l}\text { No. of measured, independent and } \\
\text { observed }[I>2 \sigma(I)] \text { reflections }\end{array}$ & $1745,441,430$ \\
\hline$R_{\text {int }}$ & 0.024 \\
\hline$(\sin \theta / \lambda)_{\max }\left(\AA^{-1}\right)$ & 0.652 \\
\hline \multicolumn{2}{|l|}{ Refinement } \\
\hline$R\left[F^{2}>2 \sigma\left(F^{2}\right)\right], w R\left(F^{2}\right), S$ & $0.019,0.048,1.23$ \\
\hline No. of reflections & 441 \\
\hline No. of parameters & 35 \\
\hline$\Delta \rho_{\max }, \Delta \rho_{\min }\left(\mathrm{e} \AA^{-3}\right)$ & $0.64,-0.74$ \\
\hline
\end{tabular}

Computer programs: APEX2 and SAINT (Bruker, 2000), SHELXS97, SHELXL97 and SHELXTL (Sheldrick, 2008) and DIAMOND (Brandenburg, 2006).

by cooling to room temperature at $20 \mathrm{~K} \mathrm{~h}^{-1}$. Colorless crystals in the millimetre range were obtained.

\section{Refinement}

Crystal data, data collection and structure refinement details are summarized in Table 2. From the two large cation positions in the structure (Wyckoff positions $3 a$ and $6 c$ ), only those of $M 1$ at $3 a$ are occupationally disordered by $\mathrm{Ba}$ and $\mathrm{Pb}$ atoms. Refinement of the occupancy of $\mathrm{Ba}: \mathrm{Pb}$ at this site under consideration of EXYZ and EADP commands (Sheldrick, 2008) resulted in a 21.6 (7)\% occupancy of $\mathrm{Pb}$. The highest peak and the deepest hole are located 0.98 and $2.06 \AA$ from the $\mathrm{Ba} 2$ and $\mathrm{B}$ atoms, respectively.

\section{Funding information}

Funding for this research was provided by: Discipline Construction Foundation of Tangshan College (award No. tsbc2013003).

\section{References}

Bartwal, K. S., Bhatt, R., Kar, S. \& Wadhawan, V. K. (2001). Mater. Sci. Eng. B, 85, 76-79.

Brandenburg, K. (2006). DIAMOND. Crystal Impact GbR, Bonn, Germany.

Bruker (2000). APEX2, SAINT and SADABS. Bruker AXS Inc., Madison, Wisconsin, USA.

Chen, C., Wang, Y., Wu, B., Wu, K., Zeng, W. \& Yu, L. (1995). Nature, 373, 322-324.

Chen, C., Wu, Y., Jiang, A., Wu, B., You, G., Li, R. \& Lin, S. (1989). J. Opt. Soc. Am. B, 6, 616-621. 
Chen, C., Wu, B., Jiang, A. \& You, G. (1985). Sci. Sin. B28, 235-241. Dong, X. Y., Jing, Q., Shi, Y. J., Yang, Z. H., Pan, S. L., Poeppelmeier, K. R., Young, J. \& Rondinelli, J. M. (2015). J. Am. Chem. Soc. 137, 9417-9422.

Huang, Y., Wu, L., Wu, X., Li, L., Chen, L. \& Zhang, Y. (2010). J. Am. Chem. Soc. 132, 12788-12789.

Jing, Q., Dong, X. Y., Yang, Z. H. \& Pan, S. L. (2015). Dalton Trans. 44, 16818-16823.

Li, H. Y., Dong, L., Lu, Y., Pan, S. L., Lu, X., Yu, H. W., Wu, H. P., Su, X. \& Yang, Z. H. (2014). J. Alloys Compd. 615, 561-565.

Liu, L. L., Yang, Y., Jing, Q., Dong, X. Y., Yang, Z. H., Pan, S. L. \& Wu, K. (2016). J. Phys. Chem. C, 120, 18763-18770.

Liu, L., Zhang, B. B., Zhang, F. F., Pan, S. L., Zhang, F. Y., Zhang, X. W., Dong, X. Y. \& Yang, Z. H. (2015). Dalton Trans. 44, 70417047.

Mutailipu, M., Hou, D. W., Zhang, M., Yang, Z. H. \& Pan, S. L. (2016). New J. Chem. 40, 6120-6126.
Sasaki, T., Mori, Y., Yoshimura, M., Yap, Y. K. \& Kamimura, T. (2000). Mater. Sci. Eng. Rep. 30, 1-54.

Sheldrick, G. M. (2008). Acta Cryst. A64, 112-122.

Tang, X. L., Feng, D. X., Wan, S. M., Kang, L., Zhang, B. \& Lin, Z. S. (2015). Mater. Chem. Phys. 163, 501-506.

Wu, H. P., Pan, S. L., Jia, D. Z., Chen, Z. H. \& Yu, H. W. (2012). Chem. Lett. 41, 812-813.

Wu, S., Wang, G., Xie, J., Wu, X., Zhang, Y. \& Lin, X. (2002). J. Cryst. Growth, 245, 84-86.

Zhang, G., Liu, Z., Zhang, J., Fan, F., Liu, Y. \& Fu, P. (2009). Cryst. Growth Des. 9, 3137-3141.

Zhang, F. F., Zhang, F. Y., Lei, B. H., Yang, Z. H. \& Pan, S. L. (2016). J. Phys. Chem. C, 120, 12757-12764.

Zhang, H., Zhang, M., Pan, S. L., Yang, Z., Wang, Z., Bian, Q., Hou, X., Yu, H., Zhang, F., Wu, K., Yang, F., Peng, Q., Xu, Z., Chang, K. B. \& Poeppelmeier, K. R. (2015). Cryst. Growth Des. 15, 523529. 


\section{supporting information}

Acta Cryst. (2017). E73, 349-353 [https://doi.org/10.1107/S2056989017001864]

Crystal structure of the solid solution $\mathrm{Ba}_{8.35} \mathrm{~Pb}_{0.65}\left(\mathrm{~B}_{3} \mathrm{O}_{6}\right)_{6}$

\section{Wenwu Zhao}

\section{Computing details}

Data collection: APEX2 (Bruker, 2000); cell refinement: SAINT (Bruker, 2000); data reduction: SAINT (Bruker, 2000); program(s) used to solve structure: SHELXS97 (Sheldrick, 2008); program(s) used to refine structure: SHELXL97 (Sheldrick, 2008); molecular graphics: DIAMOND (Brandenburg, 2006); software used to prepare material for publication: SHELXTL (Sheldrick, 2008).

Octabarium lead(II) hexakis(triborate)

Crystal data

$\mathrm{Ba}_{8.35} \mathrm{~Pb}_{0.65}\left(\mathrm{~B}_{3} \mathrm{O}_{6}\right)_{6}$

$M_{r}=2051.83$

Trigonal, $R \overline{3}$

Hall symbol: $-\mathrm{R} 3$

$a=7.206(2) \AA$

$c=18.653(11) \AA$

$V=838.7(6) \AA^{3}$

$Z=1$

$F(000)=899$

\section{Data collection}

Bruker APEXII CCD diffractometer

Radiation source: fine-focus sealed tube Graphite monochromator

phi and $\omega$ scans

Absorption correction: numerical

(face-indexed using $S A D A B S$; Bruker, 2000)

$T_{\min }=0.141, T_{\max }=0.547$

\section{Refinement}

Refinement on $F^{2}$

Least-squares matrix: full

$R\left[F^{2}>2 \sigma\left(F^{2}\right)\right]=0.019$

$w R\left(F^{2}\right)=0.048$

$S=1.23$

441 reflections

35 parameters

0 restraints

Primary atom site location: structure-invariant direct methods
$D_{\mathrm{x}}=4.062 \mathrm{Mg} \mathrm{m}^{-3}$

Mo $K \alpha$ radiation, $\lambda=0.71073 \AA$

Cell parameters from 1161 reflections

$\theta=3.3-27.6^{\circ}$

$\mu=13.00 \mathrm{~mm}^{-1}$

$T=296 \mathrm{~K}$

Plate, colourless

$0.16 \times 0.08 \times 0.02 \mathrm{~mm}$

1745 measured reflections

441 independent reflections

430 reflections with $I>2 \sigma(I)$

$R_{\text {int }}=0.024$

$\theta_{\max }=27.6^{\circ}, \theta_{\min }=3.3^{\circ}$

$h=-8 \rightarrow 9$

$k=-9 \rightarrow 9$

$l=-14 \rightarrow 23$

Secondary atom site location: difference Fourier map

$w=1 /\left[\sigma^{2}\left(F_{0}^{2}\right)+(0.0178 P)^{2}+5.852 P\right]$

where $P=\left(F_{\mathrm{o}}{ }^{2}+2 F_{\mathrm{c}}{ }^{2}\right) / 3$

$(\Delta / \sigma)_{\max }<0.001$

$\Delta \rho_{\max }=0.64 \mathrm{e} \AA^{-3}$

$\Delta \rho_{\min }=-0.74$ e $\AA^{-3}$

Extinction correction: SHELXL97 (Sheldrick, 2008), $\mathrm{Fc}^{*}=\mathrm{kFc}\left[1+0.001 \mathrm{xFc}^{2} \lambda^{3} / \sin (2 \theta)\right]^{-1 / 4}$

Extinction coefficient: 0.00125 (17) 


\section{Special details}

Geometry. All esds (except the esd in the dihedral angle between two 1.s. planes) are estimated using the full covariance matrix. The cell esds are taken into account individually in the estimation of esds in distances, angles and torsion angles; correlations between esds in cell parameters are only used when they are defined by crystal symmetry. An approximate (isotropic) treatment of cell esds is used for estimating esds involving l.s. planes.

Refinement. Refinement of $\mathrm{F}^{2}$ against ALL reflections. The weighted R-factor $\mathrm{wR}$ and goodness of fit $\mathrm{S}$ are based on $\mathrm{F}^{2}$, conventional $\mathrm{R}$-factors $\mathrm{R}$ are based on $\mathrm{F}$, with $\mathrm{F}$ set to zero for negative $\mathrm{F}^{2}$. The threshold expression of $\mathrm{F}^{2}>2 \operatorname{sigma}\left(\mathrm{F}^{2}\right)$ is used only for calculating R-factors(gt) etc. and is not relevant to the choice of reflections for refinement. R-factors based on $\mathrm{F}^{2}$ are statistically about twice as large as those based on F, and R- factors based on ALL data will be even larger.

Fractional atomic coordinates and isotropic or equivalent isotropic displacement parameters $\left(\hat{A}^{2}\right)$

\begin{tabular}{llllll}
\hline & $x$ & $y$ & $z$ & $U_{\text {iso }} * / U_{\text {eq }}$ & Occ. $(<1)$ \\
\hline Pb1 & 0.0000 & 0.0000 & 0.0000 & $0.0147(2)$ & $0.216(7)$ \\
Ba1 & 0.0000 & 0.0000 & 0.0000 & $0.0147(2)$ & $0.784(7)$ \\
$\mathrm{Ba} 2$ & 0.6667 & 0.3333 & $0.12947(2)$ & $0.01537(18)$ & \\
$\mathrm{B}$ & $0.2953(7)$ & $-0.1579(7)$ & $0.0864(3)$ & $0.0175(9)$ & \\
$\mathrm{O} 1$ & $0.2633(5)$ & $0.0063(4)$ & $0.09165(18)$ & $0.0218(6)$ & \\
$\mathrm{O} 2$ & $0.5029(5)$ & $-0.1261(5)$ & $0.08345(18)$ & $0.0234(7)$ & \\
\hline
\end{tabular}

Atomic displacement parameters $\left(\mathscr{A}^{2}\right)$

\begin{tabular}{lllllll}
\hline & $U^{11}$ & $U^{22}$ & $U^{33}$ & $U^{12}$ & $U^{13}$ & $U^{23}$ \\
\hline $\mathrm{Pb} 1$ & $0.0136(2)$ & $0.0136(2)$ & $0.0169(3)$ & $0.00680(12)$ & 0.000 & 0.000 \\
$\mathrm{Ba} 1$ & $0.0136(2)$ & $0.0136(2)$ & $0.0169(3)$ & $0.00680(12)$ & 0.000 & 0.000 \\
$\mathrm{Ba} 2$ & $0.01176(19)$ & $0.01176(19)$ & $0.0226(3)$ & $0.00588(9)$ & 0.000 & 0.000 \\
$\mathrm{~B}$ & $0.019(2)$ & $0.017(2)$ & $0.018(2)$ & $0.0103(18)$ & $-0.0009(18)$ & $-0.0002(17)$ \\
$\mathrm{O} 1$ & $0.0185(14)$ & $0.0159(13)$ & $0.0320(17)$ & $0.0093(12)$ & $-0.0011(12)$ & $-0.0022(12)$ \\
$\mathrm{O} 2$ & $0.0162(14)$ & $0.0142(13)$ & $0.0384(18)$ & $0.0066(11)$ & $0.0012(13)$ & $0.0017(13)$ \\
\hline
\end{tabular}

Geometric parameters $\left(\stackrel{A}{\circ}{ }^{\circ}\right)$

\begin{tabular}{|c|c|c|c|}
\hline$(\mathrm{Pb} / \mathrm{Ba}) 1-\mathrm{O} 1^{\mathrm{i}}$ & $2.537(3)$ & $\mathrm{Ba} 2-\mathrm{O} 1^{\mathrm{vii}}$ & $2.810(3)$ \\
\hline$(\mathrm{Pb} / \mathrm{Ba}) 1-\mathrm{O}^{\mathrm{ii}}$ & $2.537(3)$ & $\mathrm{Ba} 2-\mathrm{O} 2$ & $3.030(3)$ \\
\hline$(\mathrm{Pb} / \mathrm{Ba}) 1-\mathrm{O} 1$ & $2.537(3)$ & $\mathrm{Ba} 2-\mathrm{O} 2^{\mathrm{viii}}$ & $3.030(3)$ \\
\hline$(\mathrm{Pb} / \mathrm{Ba}) 1-\mathrm{O} 1^{\mathrm{iii}}$ & $2.537(3)$ & $\mathrm{Ba} 2-\mathrm{O} 2^{\mathrm{ix}}$ & $3.030(3)$ \\
\hline$(\mathrm{Pb} / \mathrm{Ba}) 1-\mathrm{O} 1^{\mathrm{iv}}$ & $2.537(3)$ & $\mathrm{Ba} 2-\mathrm{B}^{\mathrm{viii}}$ & $3.296(5)$ \\
\hline$(\mathrm{Pb} / \mathrm{Ba}) 1-\mathrm{O}^{\mathrm{v}}$ & $2.537(3)$ & $\mathrm{Ba} 2-\mathrm{B}^{\mathrm{ix}}$ & $3.296(5)$ \\
\hline $\mathrm{Pb} 1-\mathrm{Ba} 2^{\mathrm{vi}}$ & $3.803(2)$ & $\mathrm{Ba} 2-\mathrm{Pb} 1^{\mathrm{xii}}$ & $3.803(2)$ \\
\hline $\mathrm{Pb} 1-\mathrm{Ba} 2^{\mathrm{vii}}$ & $3.803(2)$ & $\mathrm{B}-\mathrm{O} 1$ & $1.318(5)$ \\
\hline $\mathrm{Ba} 2-\mathrm{O} 1^{\text {viii }}$ & $2.766(3)$ & $\mathrm{B}-\mathrm{O} 2$ & $1.397(5)$ \\
\hline $\mathrm{Ba} 2-\mathrm{O} 1$ & $2.766(3)$ & $\mathrm{B}-\mathrm{O} 2^{\mathrm{xiii}}$ & $1.406(5)$ \\
\hline $\mathrm{Ba} 2-\mathrm{O} 1^{\mathrm{ix}}$ & $2.766(3)$ & $\mathrm{O} 1-\mathrm{Ba} 2^{\mathrm{vii}}$ & $2.810(3)$ \\
\hline $\mathrm{Ba} 2-\mathrm{O}^{\mathrm{x}}$ & $2.810(3)$ & $\mathrm{O} 2-\mathrm{B}^{\mathrm{xiv}}$ & $1.406(5)$ \\
\hline $\mathrm{Ba} 2-\mathrm{O} 1^{\mathrm{xi}}$ & $2.810(3)$ & & \\
\hline $\mathrm{O} 1^{\mathrm{i}}-\mathrm{Pb} 1-\mathrm{O} 1^{\mathrm{ii}}$ & $100.43(11)$ & $\mathrm{O} 1^{\mathrm{xi}}-\mathrm{Ba} 2-\mathrm{O} 2^{\text {viii }}$ & $67.48(9)$ \\
\hline $\mathrm{O} 1-\mathrm{Pb} 1-\mathrm{O} 1$ & $180.00(17)$ & $\mathrm{O} 1^{\mathrm{vii}}-\mathrm{Ba} 2-\mathrm{O} 2^{\text {viii }}$ & $135.55(8)$ \\
\hline $\mathrm{O} 1 \mathrm{ii}-\mathrm{Pb} 1-\mathrm{O} 1$ & $79.57(11)$ & $\mathrm{O} 2-\mathrm{Ba} 2-\mathrm{O} 2^{\mathrm{viii}}$ & $112.31(6)$ \\
\hline
\end{tabular}




$$
\begin{aligned}
& \mathrm{O} 1^{\mathrm{i}}-\mathrm{Pb} 1-\mathrm{O} 1^{\mathrm{iii}}
\end{aligned}
$$

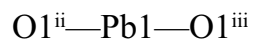

$$
\begin{aligned}
& \mathrm{O} 1-\mathrm{Pb} 1-\mathrm{O} 1^{\mathrm{iii}} \\
& \mathrm{O} 1^{\mathrm{i}}-\mathrm{Pb} 1-\mathrm{O}^{\mathrm{iv}} \\
& \mathrm{O} 1^{\mathrm{ii}}-\mathrm{Pb} 1-\mathrm{O}^{\mathrm{iv}} \\
& \mathrm{O} 1-\mathrm{Pb} 1-\mathrm{O}^{\text {iv }} \\
& \mathrm{O} 1^{\mathrm{iii}}-\mathrm{Pb} 1-\mathrm{O} 1^{\text {iv }} \\
& \mathrm{O} 1^{\mathrm{i}}-\mathrm{Pb} 1-\mathrm{O}^{\mathrm{v}} \\
& \mathrm{O} 1^{\mathrm{ii}}-\mathrm{Pb} 1-\mathrm{O}^{\mathrm{v}} \\
& \mathrm{O} 1-\mathrm{Pb} 1-\mathrm{O}^{\mathrm{v}} \\
& \mathrm{O} 1^{\mathrm{iii}-\mathrm{Pb} 1-\mathrm{O}^{\mathrm{v}}} \\
& \mathrm{O} 1^{\mathrm{iv}}-\mathrm{Pb} 1-\mathrm{O}^{\mathrm{v}} \\
& \mathrm{O} 1^{\mathrm{i}}-\mathrm{Pb} 1-\mathrm{Ba} 2^{\mathrm{vi}} \\
& \mathrm{O} 1^{\mathrm{ii}}-\mathrm{Pb} 1-\mathrm{Ba}^{\mathrm{vi}} \\
& \mathrm{O} 1-\mathrm{Pb} 1-\mathrm{Ba}^{\mathrm{vi}}
\end{aligned}
$$

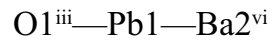

$$
\begin{aligned}
& \mathrm{O} 1^{\mathrm{iv}}-\mathrm{Pb} 1-\mathrm{Ba}^{\mathrm{vi}} \\
& \mathrm{O} 1^{\mathrm{v}}-\mathrm{Pb} 1-\mathrm{Ba}^{\mathrm{vi}} \\
& \mathrm{O} 1^{\mathrm{i}}-\mathrm{Pb} 1-\mathrm{Ba}^{\mathrm{vii}} \\
& \mathrm{O} 1^{\mathrm{ii}}-\mathrm{Pb} 1-\mathrm{Ba}^{\text {vii }} \\
& \mathrm{O} 1-\mathrm{Pb} 1-\mathrm{Ba}^{\text {vii }} \\
& \mathrm{O} 1^{\mathrm{iii}}-\mathrm{Pb} 1-\mathrm{Ba}_{2}{ }^{\mathrm{vii}} \\
& \mathrm{O} 1^{\mathrm{iv}}-\mathrm{Pb} 1-\mathrm{Ba}_{2}{ }^{\mathrm{vii}} \\
& \mathrm{O} 1^{v}-\mathrm{Pb} 1-\mathrm{Ba}^{\mathrm{vii}} \\
& \mathrm{Ba} 2^{\mathrm{vi}}-\mathrm{Pb} 1-\mathrm{Ba}^{\mathrm{vii}} \\
& \mathrm{O} 1^{\text {viii-Ba2-O1 }} \\
& \mathrm{O} 1^{\text {viii }-\mathrm{Ba} 2-\mathrm{O}^{\mathrm{ix}}} \\
& \mathrm{O} 1-\mathrm{Ba} 2-\mathrm{O}^{\text {ix }} \\
& \mathrm{O} 1^{\text {viii- }} \mathrm{Ba} 2-\mathrm{O}^{\mathrm{x}} \\
& \mathrm{O} 1-\mathrm{Ba} 2-\mathrm{O}^{\mathrm{x}} \\
& \mathrm{O} 1^{\mathrm{ix}}-\mathrm{Ba} 2-\mathrm{O} 1^{\mathrm{x}} \\
& \mathrm{O} 1{ }^{\text {viii }-\mathrm{Ba} 2-\mathrm{O}^{\mathrm{xi}}} \\
& \mathrm{O} 1-\mathrm{Ba} 2-\mathrm{O}^{\mathrm{xi}} \\
& \mathrm{O} 1^{\mathrm{ix}}-\mathrm{Ba} 2-\mathrm{O} 1^{\mathrm{xi}} \\
& \mathrm{O} 1^{\mathrm{x}}-\mathrm{Ba} 2-\mathrm{O} 1^{\mathrm{xi}}
\end{aligned}
$$

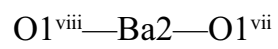

$$
\begin{aligned}
& \mathrm{O} 1-\mathrm{Ba} 2-\mathrm{O}^{\text {vii }} \\
& \mathrm{O} 1^{\mathrm{ix}}-\mathrm{Ba} 2-\mathrm{O} 1^{\mathrm{vii}} \\
& \mathrm{O} 1^{\mathrm{x}}-\mathrm{Ba} 2-\mathrm{O} 1^{\text {vii }} \\
& \mathrm{O} 1^{\mathrm{xi}}-\mathrm{Ba} 2-\mathrm{O} 1^{\mathrm{vii}} \\
& \mathrm{O} 1{ }^{\text {vii }-\mathrm{Ba} 2-\mathrm{O} 2} \\
& \mathrm{O} 1-\mathrm{Ba} 2-\mathrm{O} 2 \\
& \mathrm{O} 1{ }^{\mathrm{ix}}-\mathrm{Ba} 2-\mathrm{O} 2 \\
& \mathrm{O} 1{ }^{x}-\mathrm{Ba} 2-\mathrm{O} 2 \\
& \mathrm{O} 1{ }^{\mathrm{xi}}-\mathrm{Ba} 2-\mathrm{O} 2 \\
& \mathrm{O} 1^{\mathrm{vi}-\mathrm{Ba} 2-\mathrm{O} 2}
\end{aligned}
$$

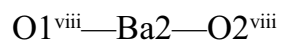

$$
\begin{aligned}
& \mathrm{O} 1-\mathrm{Ba} 2-\mathrm{O}^{\text {viii }}
\end{aligned}
$$

$$
\begin{aligned}
& \mathrm{O} 1^{\text {viii }}-\mathrm{Ba} 2-\mathrm{O} 2^{\text {ix }} \\
& \mathrm{O} 1-\mathrm{Ba} 2-\mathrm{O} 2^{\text {ix }} \\
& \mathrm{O} 1^{\mathrm{ix}}-\mathrm{Ba} 2-\mathrm{O} 2^{\mathrm{ix}} \\
& \mathrm{O} 1^{\mathrm{x}}-\mathrm{Ba} 2-\mathrm{O} 2^{\mathrm{ix}} \\
& \mathrm{O} 1^{\mathrm{xi}}-\mathrm{Ba} 2-\mathrm{O} 2^{\mathrm{ix}} \\
& \mathrm{O} 1^{\mathrm{vii}}-\mathrm{Ba} 2-\mathrm{O} 2^{\mathrm{ix}} \\
& \mathrm{O} 2-\mathrm{Ba} 2-\mathrm{O} 2^{\mathrm{ix}} \\
& \mathrm{O} 2^{\text {viii }-}-\mathrm{Ba} 2-\mathrm{O} 2^{\text {ix }} \\
& \mathrm{O} 1{ }^{\text {viii- }-\mathrm{Ba} 2-\mathrm{B}^{\text {viii }}} \\
& \mathrm{O} 1-\mathrm{Ba} 2-\mathrm{B}^{\text {viii }} \\
& \mathrm{O} 1^{\text {ix }}-\mathrm{Ba} 2-\mathrm{B}^{\text {viii }} \\
& \mathrm{O} 1^{\mathrm{x}}-\mathrm{Ba} 2-\mathrm{B}^{\mathrm{viii}} \\
& \mathrm{O} 1^{\mathrm{xi}}-\mathrm{Ba} 2-\mathrm{B}^{\text {viii }} \\
& \mathrm{O} 1^{\text {vii }-\mathrm{Ba} 2-\mathrm{B}^{\mathrm{viii}}} \\
& \mathrm{O} 2-\mathrm{Ba} 2-\mathrm{B}^{\text {viii }} \\
& \mathrm{O} 2^{\text {viii- }-\mathrm{Ba} 2-\mathrm{B}^{\text {viii }}} \\
& \mathrm{O} 2^{\mathrm{ix}}-\mathrm{Ba} 2-\mathrm{B}^{\text {viii }} \\
& \mathrm{O} 1^{\text {viii- }-\mathrm{Ba} 2-\mathrm{B}^{\mathrm{ix}}} \\
& \mathrm{O} 1-\mathrm{Ba} 2-\mathrm{B}^{\mathrm{ix}} \\
& \mathrm{O} 1^{\mathrm{ix}}-\mathrm{Ba} 2-\mathrm{B}^{\mathrm{ix}} \\
& \mathrm{O} 1{ }^{\mathrm{x}}-\mathrm{Ba} 2-\mathrm{B}^{\mathrm{ix}} \\
& \mathrm{O} 1^{\mathrm{xi}}-\mathrm{Ba} 2-\mathrm{B}^{\mathrm{ix}} \\
& \mathrm{O} 1^{\mathrm{vi}-\mathrm{Ba} 2-\mathrm{B}^{\mathrm{ix}}} \\
& \mathrm{O} 2-\mathrm{Ba} 2-\mathrm{B}^{\mathrm{ix}} \\
& \mathrm{O} 2^{\text {viii- }-\mathrm{Ba} 2-\mathrm{B}^{\mathrm{ix}}} \\
& \mathrm{O} 2^{\mathrm{ix}}-\mathrm{Ba} 2-\mathrm{B}^{\mathrm{ix}} \\
& \mathrm{B}^{\text {viii-Ba2-B }} \mathrm{B}^{\mathrm{ix}} \\
& \mathrm{O} 1^{\text {viii- }} \mathrm{Ba} 2-\mathrm{Pb}^{\mathrm{xii}} \\
& \mathrm{O} 1-\mathrm{Ba} 2-\mathrm{Pb}^{\mathrm{xii}} \\
& \mathrm{O} 1^{\mathrm{ix}}-\mathrm{Ba} 2-\mathrm{Pb} 1^{\mathrm{xii}} \\
& \mathrm{O} 1^{\mathrm{x}}-\mathrm{Ba} 2-\mathrm{Pb}^{\mathrm{xii}} \\
& \mathrm{O}^{\mathrm{xi}}-\mathrm{Ba} 2-\mathrm{Pb}^{\mathrm{xii}} \\
& \mathrm{O} 1^{\mathrm{vii}}-\mathrm{Ba} 2-\mathrm{Pb}^{\mathrm{xii}} \\
& \mathrm{O} 2-\mathrm{Ba} 2-\mathrm{Pb}^{\mathrm{xii}}
\end{aligned}
$$

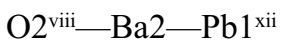

$$
\begin{aligned}
& \mathrm{O} 2^{\mathrm{ix}}-\mathrm{Ba} 2-\mathrm{Pb}^{\mathrm{xii}}
\end{aligned}
$$

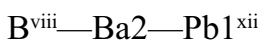

$$
\begin{aligned}
& \mathrm{B}^{\mathrm{ix}}-\mathrm{Ba} 2-\mathrm{Pb}^{\mathrm{xii}} \\
& \mathrm{O} 1-\mathrm{B}-\mathrm{O} 2 \\
& \mathrm{O} 1-\mathrm{B}-\mathrm{O} 2^{\text {xiii }} \\
& \mathrm{O} 2-\mathrm{B}-\mathrm{O} 2^{\mathrm{xiii}} \\
& \mathrm{B}-\mathrm{O} 1-\mathrm{Pb} 1 \\
& \mathrm{~B}-\mathrm{O} 1-\mathrm{Ba} 2 \\
& \mathrm{~Pb} 1-\mathrm{O} 1-\mathrm{Ba} 2 \\
& \mathrm{~B}-\mathrm{O} 1-\mathrm{Ba}^{\mathrm{vii}} \\
& \mathrm{Pb} 1-\mathrm{O} 1-\mathrm{Ba}^{\text {vii }} \\
& \mathrm{Ba} 2-\mathrm{O} 1-\mathrm{Ba} 2^{\text {vii }} \\
& \mathrm{B}-\mathrm{O} 2-\mathrm{B}^{\mathrm{xiv}}
\end{aligned}
$$

$147.14(9)$

$67.39(8)$

$47.75(8)$

$135.55(8)$

$107.59(8)$

$67.48(9)$

$112.31(6)$

$112.31(6)$

$23.05(10)$

$134.00(10)$

92.24 (10)

$89.14(10)$

75.27 (10)

$144.45(11)$

$89.88(9)$

$25.06(9)$

$134.51(10)$

$134.00(10)$

$92.24(10)$

$23.05(10)$

$144.45(11)$

89.14 (10)

$75.27(10)$

$134.51(10)$

$89.88(10)$

$25.06(9)$

$114.27(7)$

$104.78(7)$

$104.78(7)$

$104.78(7)$

$41.85(7)$

$41.85(7)$

$41.85(7)$

$106.46(7)$

$106.46(7)$

$106.46(7)$

$104.10(8)$

$104.10(8)$

120.6 (4)

$122.6(4)$

116.8 (4)

113.5 (3)

101.7 (3)

130.17 (12)

117.4 (3)

$90.51(10)$

103.73 (10)

122.9 (4) 


$\begin{array}{llll}\mathrm{O}^{1}{ }^{\mathrm{ix}}-\mathrm{Ba} 2-\mathrm{O}^{\text {viii }} & 67.39(8) & \mathrm{B}-\mathrm{O} 2-\mathrm{Ba} 2 & 88.2(2) \\ \mathrm{O} 1^{\mathrm{x}}-\mathrm{Ba} 2-\mathrm{O}^{\text {viii }} & 107.59(8) & \mathrm{B}^{\mathrm{xiv}}-\mathrm{O} 2-\mathrm{Ba} 2 & 143.4(3)\end{array}$

Symmetry codes: (i) $-x,-y,-z$; (ii) $-y, x-y, z$; (iii) $y,-x+y,-z$; (iv) $x-y, x,-z$; (v) $-x+y,-x$, $z$; (vi) $x-2 / 3, y-1 / 3, z-1 / 3$; (vii) $-x+2 / 3,-y+1 / 3,-z+1 / 3$; (viii) $-y+1, x-y, z$; (ix) $-x+y+1,-x+1, z$; (x) $y+2 / 3,-x+y+1 / 3,-z+1 / 3$; (xi) $x-y+2 / 3, x+1 / 3,-z+1 / 3$; (xii) $x+2 / 3, y+1 / 3, z+1 / 3$; (xii) $-y, x-y-1, z$; (xiv) $-x+y+1$,

$-x, z$. 